\title{
Identification of a novel agrin-dependent pathway in cell signaling and adhesion within the erythroid niche
}

\author{
A Anselmo ${ }^{1}$, E Lauranzano ${ }^{1,2}$, C Soldani ${ }^{1}$, C Ploia ${ }^{1}$, R Angioni ${ }^{2}$, G D'amico ${ }^{3}$, A Sarukhan ${ }^{4}$, C Mazzon ${ }^{\star, 12,6}$ and A Viola ${ }^{2,5,6}$
}

Establishment of cell-cell adhesion is crucial in embryonic development as well as within the stem cell niches of an adult. Adhesion between macrophages and erythroblasts is required for the formation of erythroblastic islands, specialized niches where erythroblasts proliferate and differentiate to produce red blood cells throughout life. The Eph family is the largest known family of receptor tyrosine kinases (RTKs) and controls cell adhesion, migration, invasion and morphology by modulating integrin and adhesion molecule activity and by modifying the actin cytoskeleton. Here, we identify the proteoglycan agrin as a novel regulator of Eph receptor signaling and characterize a novel mechanism controlling cell-cell adhesion and red cell development within the erythroid niche. We demonstrate that agrin induces clustering and activation of EphB1 receptors on developing erythroblasts, leading to the activation of $\alpha 5 \beta 1$ integrins. In agreement, agrin knockout mice display severe anemia owing to defective adhesion to macrophages and impaired maturation of erythroid cells. These results position agrin-EphB1 as a novel key signaling couple regulating cell adhesion and erythropoiesis.

Cell Death and Differentiation (2016) 23, 1322-1330; doi:10.1038/cdd.2016.10; published online 18 March 2016

During embryonic development, adhesive interactions trigger a variety of morphogenetic processes throughout the organism, involving either direct cell-cell contact or adhesion to extracellular matrix networks. Eph receptors form the largest subfamily of receptor tyrosine kinases (RTKs) and, together with their ligands ephrins, control cell adhesion, migration, invasion and morphology by influencing integrin and intercellular adhesion molecule activity and by modification of actin cytoskeleton organization. ${ }^{1,2}$ Eph/ephrin molecules are involved in a wide array of developmental processes such as cardiovascular and skeletal development, axon guidance and tissue patterning. ${ }^{3}$ Although Eph/ephrins have been studied classically in a developmental context, their physiological functions in the adult came to light, as they have been implicated in bone homeostasis, ${ }^{4}$ in red blood cell differentiation, ${ }^{5}$ and in alterations that lead to congenital diseases and cancer. ${ }^{6}$

Erythropoiesis, the process by which red blood cells are produced, is pivotal during all stages of life to all the vertebrates' healthy existence. In adults, red blood cells are the terminally differentiated, end-product cells of a complex hierarchy of hematopoietic progenitors that become progressively restricted to the erythroid lineage. ${ }^{7}$ Mouse erythropoiesis takes place in the fetal liver between embryonic days 12.5 and 15.5, in the neonatal spleen, and in both spleen and bone marrow of adults. Definitive erythropoiesis takes place in specialized niches, the erythoblastic islands (EI), ${ }^{8}$ consisting of a central macrophage that extends cytoplasmic protrusions to a ring of surrounding erythroid precursors in different stages of maturation. It has been proposed that El allow macrophages to provide nutrients and survival signals to the erythroblasts, to promote enucleation and to phagocytize the extruded erythroblast nuclei. ${ }^{9,10}$ Recent advances in the understanding of the interactions within the erythroid niches have pointed out the extraordinary complexity of the adhesive interaction system that involves a wide range of ligands, such as $\beta 1$ integrin, CD44, Lu and ICAM-4. ${ }^{11-13}$

Agrin is an extracellular matrix protein belonging to the heterogeneous family of heparan sulfate proteoglycans. Its best-characterized role is in the development of the neuromuscular junction during embryogenesis where it is expressed by motor neurons and leads to clustering and activation of the RTK MuSK. ${ }^{14}$ However, agrin is expressed in many tissues and its non-neuronal functions are poorly understood. We previously demonstrated that in the hematopoietic compartment agrin signals through the dystroglycan (DG) receptor by activating the Grb2-SOS-Ras signaling pathway ${ }^{15,16}$ required for survival and differentiation of hematopoietic stem cells.

In the present work, we have addressed the role of agrin in erythropoiesis. We provide evidence for a novel mechanism, mediated by the agrin-DG signaling pathway, regulating the fitness of the erythroid niche.

\footnotetext{
${ }^{1}$ Humanitas Clinical and Research Centre, Rozzano (Milan), Italy; ${ }^{2}$ Biomedical Sciences Department, University of Padua, Padua, Italy; ${ }^{3}$ Centro Ricerca Tettamanti, Department of Pediatrics, University of Milano-Bicocca, Fondazione MBBM/San Gerardo Hospital, Monza, Italy; ${ }^{4}$ INSERM, Paris, France and ${ }^{5}$ Venetian Institute of Molecular Medicine, Padova (VIMM), Italy

*Corresponding author: C Mazzon, Biomedical Sciences Department, University of Padua, via Ugo Bassi 58 B, Padua 35131, Italy. Tel: +39 049 7923 274; Fax: +39 049 7923 250; E-mail: cristina.mazzon@unipd.it

${ }^{6}$ These authors contributed equally to this work.

Abbreviations: Agrn, agrin; BM, bone marrow; BMT, bone marrow transfer; DG, dystroglycan; El, erythoblastic island; Eph, erythropoietin-producing human hepatocellular carcinoma line; Ephrin, Eph family receptor interacting proteins; GAPDH, glyceraldehyde 3-phosphate dehydrogenase; Grb2, growth factor receptor-bound protein 2; ICAM-4, intercellular adhesion molecule 4; Lrp4, lipoprotein-related receptor 4; LU, lutheran glycoprotein; MEP, megakaryocyte erythroid progenitors; MuSK, muscle-specific kinase; PBS, phosphate-buffered saline; PCR, polymerase chain reaction; RAS, rat sarcoma; RTK, receptor tyrosine kinases; SOS, son of sevenless guanine nucleotide exchange factor; VCAM-1, vascular cell adhesion molecule 1

Received 30.10.15; revised 30.12.15; accepted 05.1.16; Edited by RA Knight; published online 18.3.16
} 


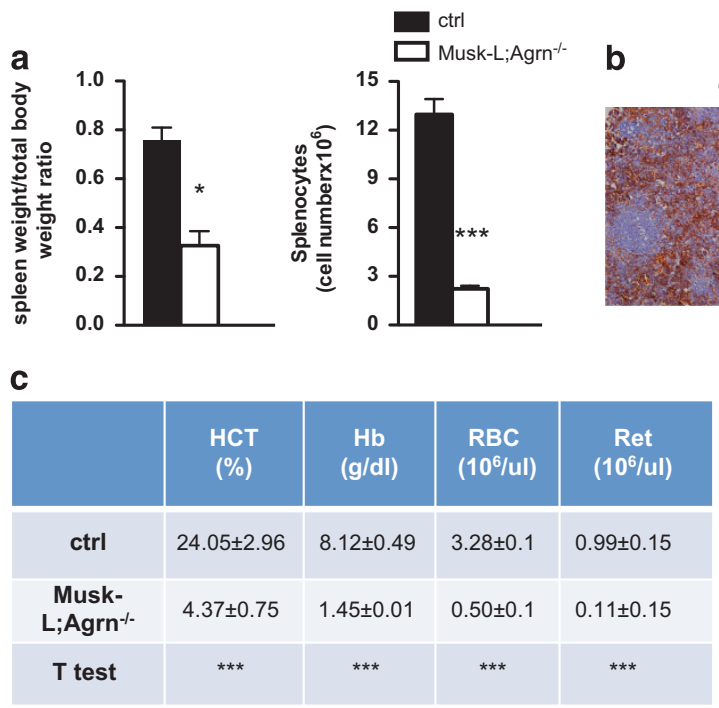

Figure 1 Erythropoietic defects in agrin-deficient mice. (a) Musk-L;Agrn ${ }^{-1-}$ ratio of the spleen weight/total body weight (left) and total splenocyte mean numbers relative to controls (right) at postnatal day 5 (P5) are shown. (ctrl: $n=19$, left;, $n=12$,right; Musk-L;Agrn ${ }^{-1-}: n=10$, left; $n=7$, right). In all panels, error bars represent S.E.M. (b) Representative immunohistochemical analysis of TER-119 on spleen sections of P5 control and agrin-deficient mice. Scale bar, $100 \mu \mathrm{m}$ (c) Whole blood of control and MuskL;Agrn ${ }^{-1-}$ mice was analyzed using an automated hematology analyzer (ctrl, $n=7$; Musk-L;Agrn ${ }^{-1-}, n=3$ ). In all panels, error bars represent S.E.M. and ${ }^{*} P \leqslant 0.05$, ${ }^{* \star *} P \leqslant 0.0001$

\section{Results}

Agrin knockout mice display an anemic phenotype. Mice lacking a functional agrin gene display embryonic lethality because of neuromuscular synapse dysfunctions, thus strongly limiting the studies in agrin-deficient adult animals. ${ }^{17}$ To overcome this limitation, in our previous ${ }^{15,16}$ and present studies, we took advantage of the Musk-L; $\mathrm{Agrn}^{-1-}$ mouse model, null for all agrin isoforms but carrying a transgene that increases Musk expression in skeletal muscle. $^{18}$ Musk-L;Agrn ${ }^{-1-}$ mice live longer, but still die postnatally between day (P) 7 and P10 for unknown reasons $^{18}$ and our experiments were therefore performed with P5 mice. Musk-L;Agrn ${ }^{+/+}$or Musk-L;Agrn ${ }^{+/-}$littermates (referred to as control mice) did not differ in any of the analyzed phenotypes, ${ }^{15}$ and hence, were not further distinguished. As already described, ${ }^{15}$ P5 Musk-L;Agrn ${ }^{-1-}$ mice (referred to hereafter as agrin-deficient mice) are characterized by smaller size than control littermates, with a reduced number of hematopoietic cells in bone marrow, spleen and blood. In addition, agrin-deficient mice showed major alterations in the spleen, which is an important site of extramedullary hematopoiesis in neonatal mice and contains a large percentage of proliferating cells, among which about $50 \%$ are erythroblasts. Agrin-deficient mice showed a significant reduction in the spleen-to-body weight ratio and in the total number of splenocytes (Figure 1a). The immunohistochemical analysis of the erythroid-specific glycophorin-A-associated Ter-119 marker on spleen sections showed a significant reduction of the red pulp in agrindeficient mice as compared with their control littermates (Figure 1b). In agreement, blood analyses indicated that hematocrit, hemoglobin, red blood cell and reticulocyte count indices were strongly reduced in agrin-deficient mice
(Figure 1c). These results indicate that erythropoiesis in agrin-deficient mice is severely impaired.

Cell-autonomous control of late erythropoiesis by agrin. In agreement with our previous study showing bone marrow hypoplasia, as well as a reduction of the $\mathrm{Lin}^{-} \mathrm{IL} 7 \mathrm{R}^{-} \mathrm{C}-\mathrm{Kit}^{+} \mathrm{Sca}-$ $1^{+}$(LSK) HSC population, ${ }^{15}$ the analysis of bone marrow progenitors showed that both frequency and absolute number of megakaryocyte erythroid progenitors (MEP) were reduced in agrin-deficient (Musk-L;Agrn ${ }^{-1}$ ) mice as compared with control littermates (Supplementary Figure 1A). This drop was paralleled by increased apoptosis in agrin-deficient MEP (Supplementary Figure 1B), suggesting a role for agrin and/or its receptor in promoting MEP survival. Indeed, we found that MEP progenitors express both agrin and its hematopoietic receptor $a$-DG, ${ }^{15,16}$ as detected by flow cytometry analysis (Supplementary Figure 1C).

We then examined whether agrin deficiency affected the different stages of the erythroid maturation process. On the basis of the dynamic changes in membrane protein expression that characterize each erythroid differentiation step, we performed flow cytometry analysis with the erythroid-specific Ter-119 and the non-erythroid-specific transferrin receptor CD71 markers ${ }^{13,19,20}$ that, together, identify four subpopulations (R1-R4) representing sequential stages of maturation: proerythroblasts and early basophilic erythroblasts (R1); early and late basophilic erythroblasts (R2); chromatophilic and orthochromatophilic erythroblasts (R3); and late orthochromatophilic erythroblasts and reticulocytes (R4) (Figure 2a). Similar results were obtained using the double staining CD44/TER-119 21 (not shown). Confocal microscopy and flow cytometry were used to validate the expression of agrin and its ligand $a$-DG ${ }^{15,16}$ in control R1-R4 subsets; the results indicate that both the molecules are expressed in R1-R2 subsets (Figure 2a); real-time PCR analysis confirmed that agrin is 

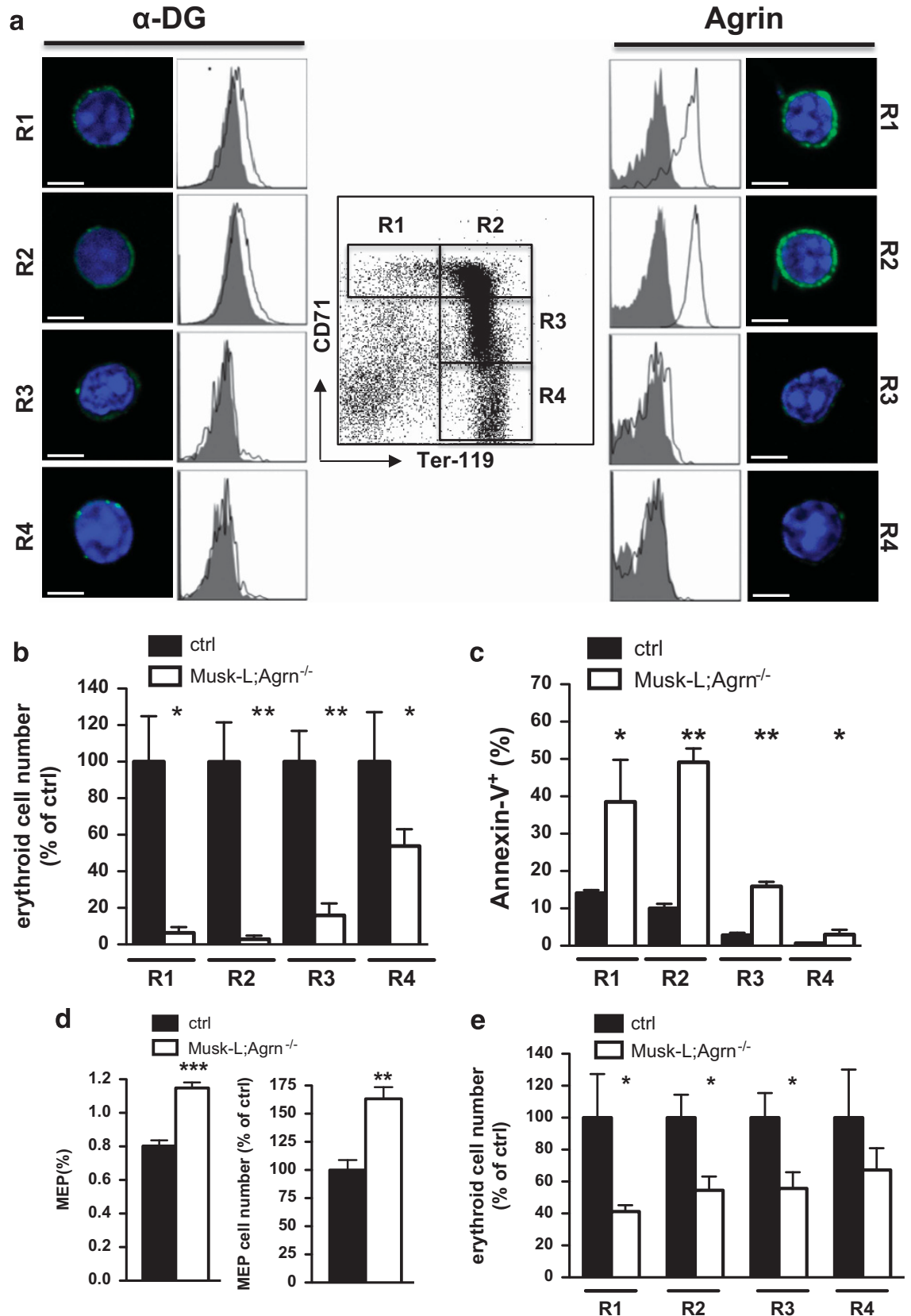

Figure 2 Agrin is required for the maturation and survival of erythroid cells. (a) Representative flow cytometry analysis and confocal images of agrin and $\alpha$-DG expression on the R1-R4 splenic erythroid subsets assessed by TER-119 and CD71 immunostaining. For flow cytometry experiments, the gray solid curve represents the isotype control. For confocal microscopy experiments, representative images are shown for a total of 30 cells analyzed for each gate (agrin or $\alpha$-DG, green; nuclear DNA, blue; scale bar, $5 \mu \mathrm{m}$ ). (b) Total cell number in each splenic erythroid subset, as determined by flow cytometry, is expressed relative to controls (ctrl, $n=10$; Musk-L;Agrn ${ }^{-1-}, n=7$ ). (c) Flow cytometry analysis of Annexin-V staining in splenic erythroid subsets of control and agrin-deficient mice (ctrl, $n=10$; Musk-L; Agrn $^{-l}{ }^{-}, n=7$ ). (d, e) Total bone marrow cells from either control or Musk-L;Agrn ${ }^{-1-}$ P5, CD45.2 mice were transplanted into lethally irradiated CD45.1 recipients. After 9 weeks, the bone marrows of transplanted mice were analyzed by flow cytometry as specified: (d) MEP frequency among total bone marrow cells (left) and mean numbers relative to controls (right) (ctrl, $n=4 ;$ Musk-L;Agrn ${ }^{\prime-}, n=8$ ); (e) Erythroid subsets (R1-R4) analyzed by TER-119 and CD71 expression (mean numbers relative to controls; $n=7$ ). In (b-e) panels, error bars represent S.E.M. and ${ }^{*} P \leqslant 0.05$, ${ }^{* \star} P \leqslant 0.01,{ }^{* \star} P \leqslant 0.0001$

endogenously produced by R1-R3 erythroid cell subsets (Supplementary Figure 2). Then, we analyzed the R1-R4 erythroid populations in agrin-deficient mice (Figure $2 \mathrm{~b}$ ). Interestingly, as compared with their littermate controls, agrin-deficient mice exhibited a reduction in the cell number of all R1-R4 erythroid cells in the spleen, although the defect was particularly dramatic in the case of the $R 2$ subset $\left(100.00 \pm 24,92\right.$ ctrl; 6,38 \pm 3.11 Musk-L;Agrn $\left.{ }^{-1-}\right)$ (Figure $2 b$ ). The reduction in cell number was accompanied by a significant increase in ex vivo apoptosis observed in the erythroid cells of agrin-deficient mice as compared with control ones (Figure 2c), whereas CD44 expression and cell size 
(expressed as FSC MFI) were similar in the two groups (Supplementary Figures $3 \mathrm{~A}$ and $3 \mathrm{~B}$ ), suggesting that a reduced cell viability was responsible for the observed decline of the erythroid populations.

Agrin-deficient mice might have multiple defects that could indirectly cause the observed impairment of erythroid cell development. ${ }^{16}$ Moreover, the analysis of cell populations in agrin-deficient mice does not allow distinguishing between a cell-autonomous or niche-derived mechanism responsible for the defective erythropoiesis in the absence of agrin. This hypothesis can only be tested performing BM transfer experiments. Lethally irradiated wild-type recipients (CD45.1) were reconstituted using total bone marrow cells from either agrin-deficient or control mice (CD45.2), and the frequency of erythroid subsets was analyzed after 9 weeks. The frequency of peripheral blood hematopoietic cells expressing CD45.2 was quantified by flow cytometry, demonstrating comparable reconstitution in all recipients, that is, $>90 \%$ for mice that received either control or Musk-L;Agrn ${ }^{-1-}$ bone marrow cells (not shown and Mazzon et al. ${ }^{15}$ ). In an agrin-sufficient stromal niche ${ }^{15}$ the generation of agrin-deficient MEP progenitors was not impaired and, interestingly, an increase in MEP numbers was detected in wild-type recipients reconstituted with agrindeficient bone marrow cells (Figure 2d). We thus assessed the in vitro culture growth of P5 erythroid progenitors in burstforming unit erythroid colony assay. In agreement with the in vivo analysis, the results showed no significant differences between control and agrin-deficient MEP (data not shown).

Whereas the experiments described above (Figure 2d and Supplementary Figure 4) demonstrated a niche-dependent role of agrin in MEP homeostasis, the analysis of agrindeficient erythroid subsets performed in the BMT recipients showed a persistent defect in the cell number as compared with the control counterpart (Figure 2e). These results support a cell-autonomous role of agrin in the control of late erythroid maturation but not of erythroid precursors.

Agrin regulates adhesive interactions at the erythroblastic islands. The observed reduced viability of agrin-deficient erythroid cells might be explained by a defective formation of $\mathrm{El}$ in the absence of agrin. Native El, isolated ex vivo from spleens of agrin-deficient or control mice (Figure 3a), as well as islands reconstituted in vitro from bone marrow cells (Figure $3 b$ ), were analyzed by confocal microscopy using the erythroid-specific TER-119 and the macrophage-specific F4/80 markers. Agrin-deficient islands exhibited a very low number of erythroid cells per macrophage, suggesting an important role of this molecule in the formation of the niche. Moreover, co-cultures of R2 cells isolated from either control or Musk-L;Agrn ${ }^{-/}$mice with control $\mathrm{F} 4 / 80^{+}$macrophages demonstrated that agrin deficiency affects red cell viability, as shown by the significantly higher number of annexin- $\mathrm{V}^{+} / \mathrm{Ter} 19^{+}$cells among agrin-deficient erythroid cells (Supplementary Figure 4). Agrin is also expressed by macrophages $^{16}$ and the dysfunctional state of agrin-deficient macrophages has already been demonstrated. ${ }^{16}$ Thus, we analyzed El reconstituted using either agrin-deficient macrophages and control erythroblasts or control macrophages and agrin-deficient erythroblasts (Figure $3 \mathrm{c}$ ). The results indicated that, in order to establish proper adhesive interactions that are required to sustain erythroblast viability, both cell types must express agrin.

We have previously demonstrated that in the hematopoietic compartment agrin signals through the DG complex. ${ }^{15,16}$ Thus, we speculated that inhibition of agrin-DG interactions would result in defective El formation. Using an antibody that blocks the interaction of agrin with the $a-\mathrm{DG}$ receptor, ${ }^{15,16}$ we inhibited El formation in cells from control mice (Figure 3d), confirming the data obtained with agrin-deficient cells. Again, the pre-incubation of either macrophages or erythroblasts with the blocking antibody resulted in impaired formation of El, indicating that both cell types require agrin and $a$-DG receptor for optimal cell-cell adhesion at the erythroid niche (Figure 3e). However, the results also indicate that, for the stability of the $\mathrm{El}$, the agrin-DG signaling is more critical for erythroid cells than macrophages.

Agrin signaling through DG results in EphB1 RTK activation. At the neuromuscular junction, agrin binds to Lipoprotein-related receptor 4 (Lrp4) and thus mediates triggering of the RTK MuSK. ${ }^{18,22}$ We therefore asked whether the interaction of agrin with DG at the El results in triggering of a member of the RTK family. R2 erythroid cells, unstimulated or stimulated for 5 and 30 min with agrin, were analyzed using a phospho-RTK array, which simultaneously determines relative phosphorylation levels in over 39 different kinases. Analysis of the subsequent phosphorylation profiles revealed that agrin stimulation induces the activation of EphB1 tyrosine kinase that depends on agrin-DG interaction (Figure 4a). This result was confirmed by western blot analysis of $\mathrm{R} 2$ erythroid cell lysates with a specific antibody against the phosphorylated form of EphB1 (Supplementary Figure 5). Eph receptor activation and signaling relies on clustering. ${ }^{6}$ Tyrosine phosphorylation of Eph receptors requires presentation of ephrin ligands in either clustered or membrane-attached forms, ${ }^{23}$ and specific Eph responses occur only at higher ligand oligomerization states. ${ }^{24}$ However, some studies suggested that Eph receptor activation is not completely explained by the model described above and indicated that ligand binding may not be required for receptor clustering and phosphorylation. ${ }^{25,26}$ We therefore asked whether agrin induced EphB1 clustering in erythroblasts. To this end, R2 cells isolated from control mice were stimulated with agrin for $10 \mathrm{~min}$, stained with anti-EphB1 antibodies and analyzed by confocal microscopy. Agrin stimulation induced EphB1 receptor capping $(77.9 \pm 12.7 \%$ of $\mathrm{R} 2$ primary cells showing EphB1 receptor clustering, Figure 4b), which was completely abrogated by pre-incubation of $\mathrm{R} 2$ cells with the agrin-DG blocking antibody $(77.9 \pm 12.7 \%$ of $\mathrm{R} 2$ primary cells showing EphB1 receptor clustering versus $13.8 \pm 1.6 \%$, Figure 4b). In agreement, agrin stimulation induced tyrosine phosphorylation of $\beta$-DG in the K562 human erythromyeloblastoid leukemia cell line (Supplementary Figure 6), as already described in neurons, ${ }^{27}$ as well as DG-mediated actin polymerization in R2 primary cells (Figure 4c) and co-capping of the phosphorylated $\beta$-DG with EphB1 at the R2 cell surface (Pearson's coefficient $=0.76 \pm 0.1, \quad P=0.0003$; Figure 4d). The physical interaction between DG and EphB1 was confirmed by co-immunoprecipitation experiments in the K562 cell line (Figure 4e). 

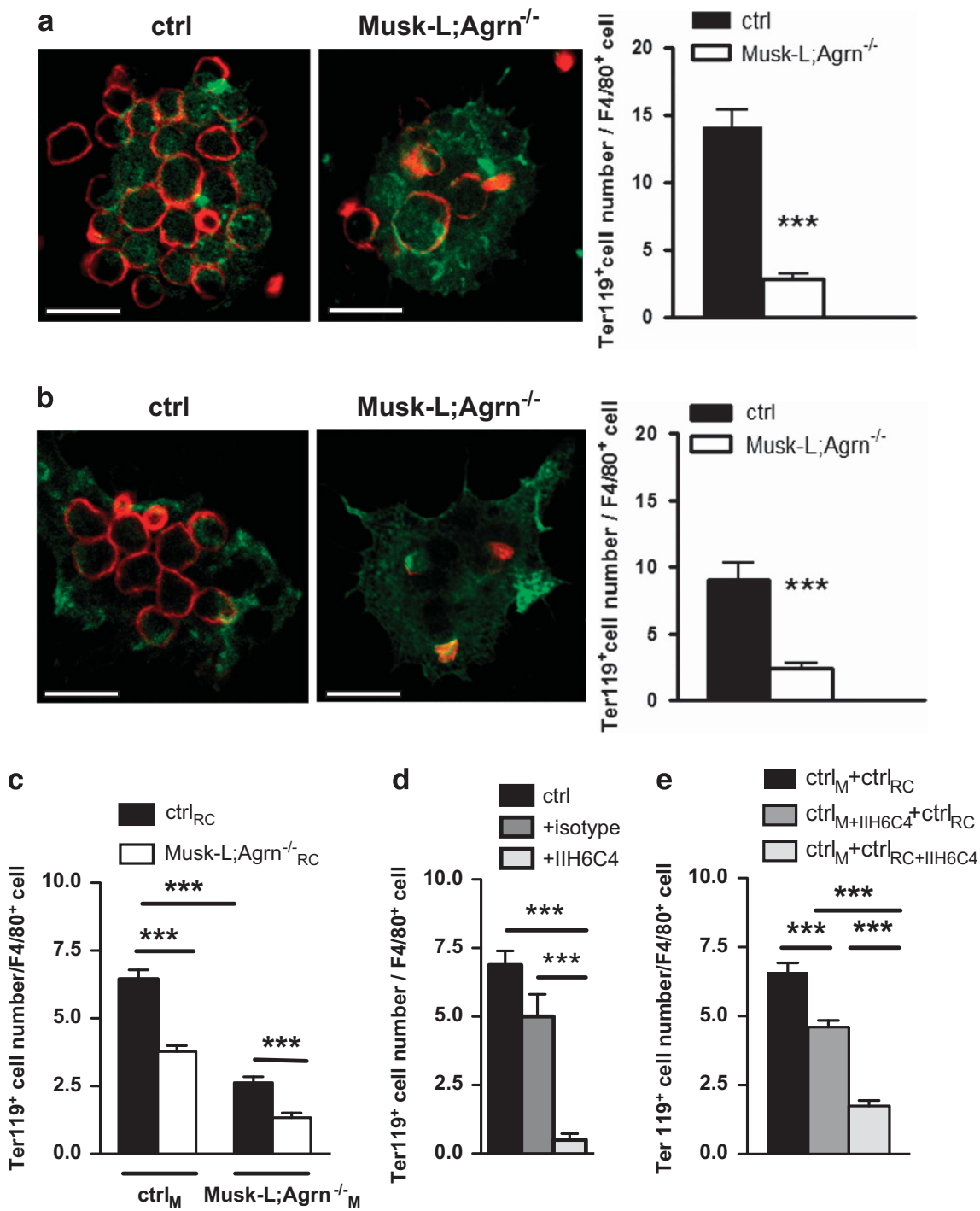

Figure 3 Agrin orchestrates cell-cell interactions at the El. (a, b) Analysis of erythroblastic islands (EI) in P5 control and agrin-deficient mice. Representative

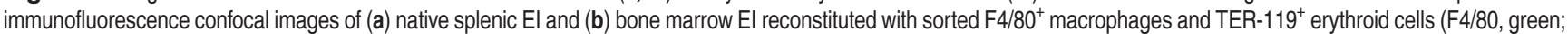
TER-119, red; scale bar $20 \mu \mathrm{m}$ ). Data shown are the mean of three pooled experiments (ctrl and Musk-L;Agrn ${ }^{-1}, n=3$ ). (c, d, e) Analysis of reconstituted El. (c) El

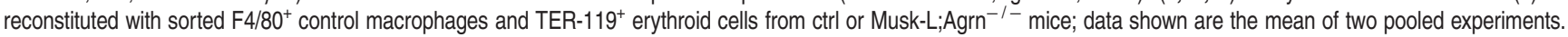

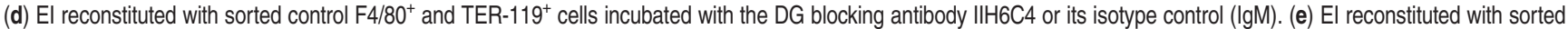

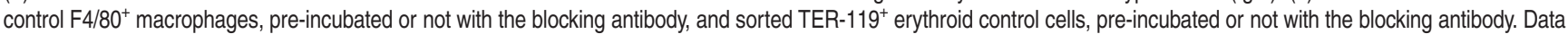
shown are the mean of two pooled experiments. In all panels (a-e), the number of TER-119+ cells per macrophage is indicated, error bars represent S.E.M. and ${ }^{* \star *} P \leqslant 0.0001$

The EphB1 downstream response is known to activate av $\beta 3$ and $a 5 \beta 1$ integrins and thus promote cell adhesion. ${ }^{28} \mathrm{We}$ therefore examined whether agrin-induced triggering of EphB1 might result in the activation of $a v \beta 3$ and $a 5 \beta 1$ integrins. Agrin stimulation of R2 erythroblasts induced the expression of both $a 5$ and $\beta 1$ integrins on the cell surface, and phosphorylation of $\beta 1$ (Figure 4f). In contrast, no statistically significant difference was observed for $\alpha \mathrm{v}$ and $\beta 3$ integrins (Supplementary Figure 7).

\section{Discussion}

Erythrocytes, produced through a process named erythropoiesis, are pivotal for the existence of all vertebrates and, indeed, agrin-deficient mice displayed overt and severe signs of anemia, with significant reductions in hematocrit, hemoglobin content and circulating red blood cells. However, no defects in fetal erythropoiesis (E15 and E17) were detected (not shown), indicating that agrin is specifically required for the regulation of postnatal erythroid development. These data are consistent with our previous report ${ }^{15}$ showing that the hematopoietic stem cell dysfunctions caused by agrin deficiency are restricted to postnatal life. The reason why agrin is specifically required for adult hematopoiesis and erythropoiesis is not known, but we speculate that other molecules may substitute agrin in the fetal liver microenvironment. The expression of both agrin and its receptor $a$-DG in red cells seems to be developmentally regulated and this is 
a

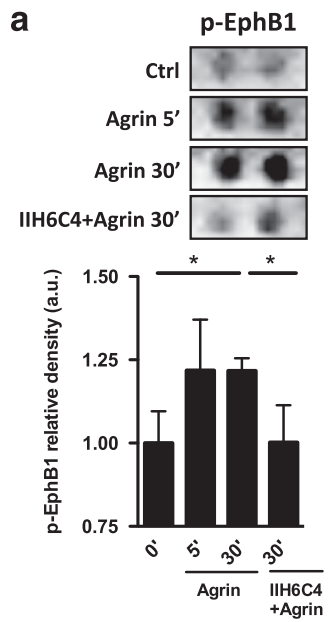

C

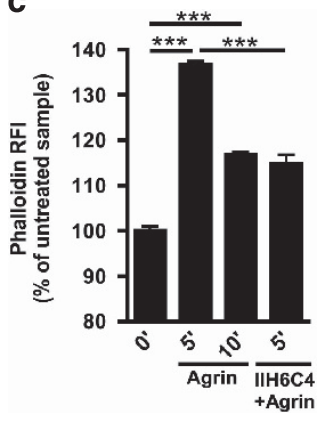

e

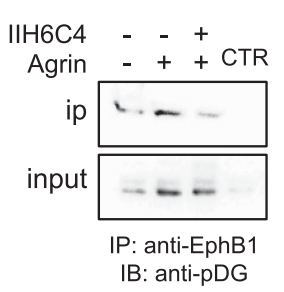

b

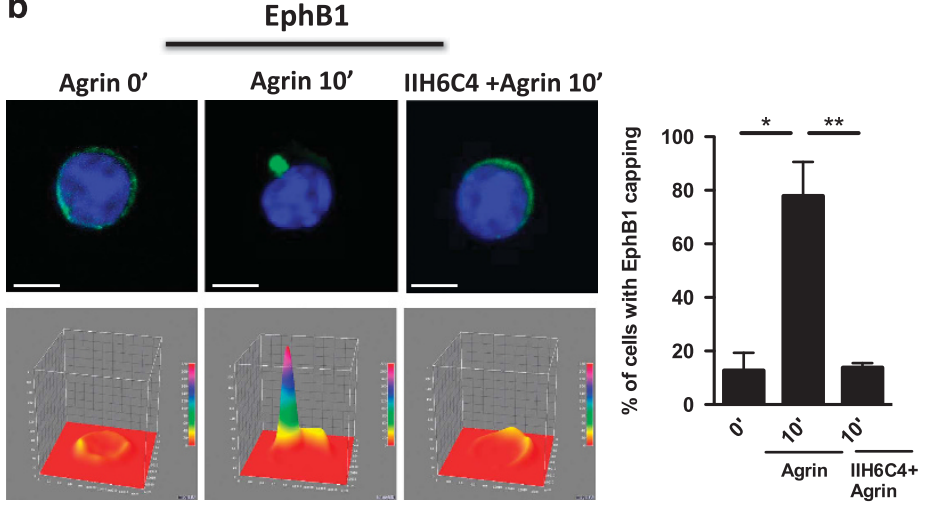

d

DAPI

DG

EphB1
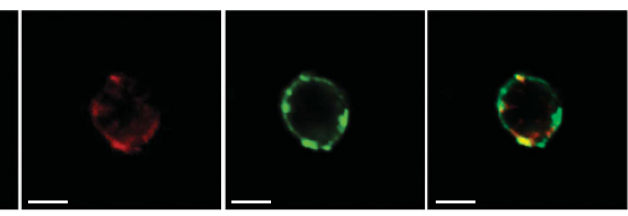

훅
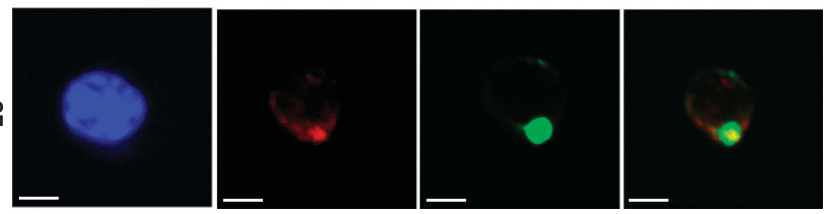

f
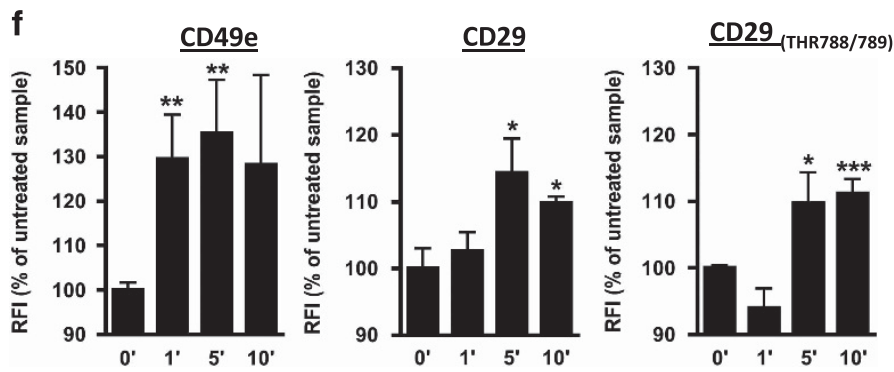

Figure 4 Agrin induces EphB1 phosphorylation in a DG-dependent manner. For experiments (a-d), primary sorted R2 erythroid cells from P5 ctrl mice were pre-incubated or not with the DG blocking antibody IIH6C4 $(10 \mu \mathrm{g} / \mathrm{ml})$ and stimulated with agrin $(10 \mu \mathrm{g} / \mathrm{ml})$ for the indicated time points. (a) EphB1 phosphorylation was determined using the mouse phosphor-RTK assay as described in methods. Histograms represent the fold increase in signal relative to unstimulated cells, and represent the mean of three pooled experiments. (b) Cell surface distribution of EphB1 was determined by staining with anti-EphB1 antibody (green) and DAPI (blue) and confocal microscopy analysis. Representative images and 3D surface plots are shown, scale bar, $5 \mu \mathrm{m}$. The percentage ( \pm S.E.M.) of cells showing EphB1 capping was calculated (at least 30 cells from three independent experiments). (c) Induction of actin polymerization (phalloidin staining) was analyzed by flow cytometry. Results are reported as fold increase of the mean fluorescence intensity (MFI) of untreated samples. (d) Colocalization of EphB1 and DG was determined by staining with DG (red), EphB1 (green) and DAPI (blue), and corresponding images were merged (yellow). Shown are representative confocal images (out of 30 cells); scale bar, $5 \mu \mathrm{m}$. (e) K562 human erythromyeloblastoid cells preincubated or not with the IIH6C4 blocking antibody and stimulated for $10 \mathrm{~min}$ with agrin were lysed and the samples immunoprecipitated and subjected to WB analysis with the indicated antibodies; one representative experiment out of three is shown. (f) Flow cytometry analysis of CD29, CD49 membrane and CD29 (THR788/789) expression on the R2 erythroid subsets from P5 spleen of control mice stimulated for 1-5-10 min with agrin. Results are reported as fold increase of the MFI of untreated samples. Data shown are the mean of four pooled experiments. In all panels, error bars represent S.E.M. and ${ }^{\star} P \leqslant 0.05,{ }^{* \star} P \leqslant 0.01$ and ${ }^{* \star \star} P \leqslant 0.0001$

consistent with the modulation of expression of adhesion molecules observed during maturation of erythroid cells. ${ }^{21}$ The reconstitution experiments performed in this study demonstrate that, even in a permissive niche, agrin-deficient hematopoietic cells display defective erythroid maturation, thus highlighting an important and novel cell-autonomous role of agrin in erythropoiesis. Consistently, both agrin and its receptor $a$-DG are expressed on erythroid subsets during erythropoiesis. Although $a$-DG expression appears similar at all maturation stages analyzed, in the case of agrin, changes in mRNA and membrane-bound protein do not correlate. This may depend on more than one reason: (i) in $\mathrm{R} 1$ cells the 
membrane-bound agrin may derive from the extracellular microenvironment; (ii) mRNA processing and stability as well as protein stability and modifications may be responsible for the different results observed by analyzing mRNA and protein expression; (iii) finally, we cannot formally exclude the presence in erythroid cells of agrin isoforms not detected by our primers. The fluctuations in membrane-bound agrin expression during the erythropoiesis process are consistent with a previous study ${ }^{21}$ showing modulation of adhesion molecule expression during the maturation of erythroid cells.

Growing evidence supports the concept of El as microenvironmental niches where cell-cell adhesive interactions, in concert with cytokines, are crucial for terminal erythroid differentiation and regulation of apoptosis. ${ }^{8,29}$ Recent advances in the understanding of the cellular and molecular interactions within the erythroid niches have pointed out the extraordinary complexity of the adhesive interaction system, where both homophilic interactions, such as those mediated by the erythroblast macrophage protein (Emp), ${ }^{30}$ as well as heterophilic ones, involving, for example, $a 4 \beta 1$ integrin and VCAM- $1,{ }^{31}$ are critical for island integrity ${ }^{13}$ although the precise roles of these adhesive connections are still unclear. ${ }^{9}$ Here, we demonstrate for the first time that agrin induces EphB1 phosphorylation in the primary R2 erythroid subset through its interaction with the $a$-DG receptor. We show that EphB1 and DG are physically and functionally associated, and provide evidence for a key role of agrin in the subsequent $a 5 \beta 1$ integrin activation, thus mediating the stability of the interaction between erythroblasts and fibronectin-expressing macrophages. Our findings are in agreement with a previous study that has suggested $a 5 \beta 1$ integrin as component or modulators of the agrin signal transduction pathway. ${ }^{32}$ We have recently reported severe defects in development and functions of agrin-deficient monocytes ${ }^{16}$ and thus we speculated that the observed defects in red cell maturation might depend on the inability of agrin-deficient macrophages to support El formation. Indeed, El isolated from agrin-deficient mice were largely defective and the in vitro experiments confirmed a role for the agrin/DG axis in their formation. Interestingly, our experiments showed that both macrophages and red cells require agrin and its receptor for optimal $\mathrm{El}$ functionality. In line with our findings, previous studies showing that the severe macrophage depletion causes a mild deficit in red cell production. ${ }^{19,20}$

Overall, our results identify new crucial players at the erythroid niche controlling adhesion in a complex manner: the DG complex binds agrin expressed by both erythroid cells and macrophages, induces clustering and activation of the RTK EphB1 and leads to the upregulation of $\alpha 5 \beta 1$ integrins. More importantly, this study demonstrates a new role for agrin and DG as triggers of EphB1 activity and suggests that similar signaling processes may occur during different cell-cell interactions and may be crucial for the activity of the Eph family kinases. Whether agrin regulates Eph signaling at the neuromuscolar junction is a question that deserves investigation.

\section{Methods}

Mice. Agrin-deficient, Musk-L;Agrn ${ }^{-1}$ mice (on C57BI/6 background) have been described elsewhere. ${ }^{15,16,18}$ Congenic (CD45.1) mice were used as recipients of bone marrow transplantation experiments. Procedures involving animals and their care conformed to institutional guidelines in compliance with national (4D.L. N.116, G.U., suppl. 40, 18-2-1992) and international (EEC Council Directive 86/609, OJ L 358,1,12-12-1987; National Institutes of Health Guide for the Care and Use of Laboratory Animals) law and policies. The protocol was approved by the Italian Ministry of Health on 18 June 2007 and modified by Protocol 162/2011-B. All efforts were made to minimize the number of animals used and their suffering.

Immunohistochemistry and immunofluorescence. Formalin-fixed paraffin-embedded 2- $\mu \mathrm{m}$ sections were deparaffinized, rehydrated and incubated after antigen retrieval with anti-TER-119 (BD Pharmingen, San Diego, CA, USA) and the appropriate secondary antibody. Staining was visualized by $3,3^{\prime}-$ diaminobenzidine (Dako, Carpinteria, CA, USA) and counterstained with hematoxylin. Images were acquired with Olympus BX51 microscope (Center Valley, PA, USA). Sorted erythroid cells were fixed with 1 or $4 \%$ formaldehyde, blocked with bovine serum albumin $1 \%$ and incubated with the primary antibodies anti-agrin (gift from Prof M. Ferns, Department of Physiology and Membrane Biology, University of California, Davis School of Medicine, Davis, CA, USA), anti- $\alpha$-DG (AbCam, Cambridge, UK), anti ph-DG (AbCam) and anti-EphB1 (M19; Santa Cruz Biotechnology, Dallas, TX, USA). To quantify EphB1 clusters, primary erythroid R2 cells, either untreated or anti- $\alpha-\mathrm{DG}$ antibody $I \mathrm{H} 6 \mathrm{CC} 4(10 \mathrm{ug} / \mathrm{ml}$, Millipore, Billerica, MA, USA) treated, were incubated with $10 \mathrm{ug} / \mathrm{ml}$ recombinant agrin (R\&D Biosystems, Minneapolis, MN, USA); after fixation in 4\% PFA for 30 min, cells were incubated with anti-EphB1 (M19; Santa Cruz) and anti-goat Alexa-conjugated secondary antibody (Molecular Probes, Eugene, OR, USA). The nuclei were counterstained with Hoechst 33342 (Molecular Probes). Images were acquired by a confocal microscope Fluoview FV1000 (Olympus) with an oil immersion objective ( $\times 40$ or $60 \times 1.4$ NA Plan-Apochromat; Olympus) using laser excitation at 405,488 , 594 or $633 \mathrm{~nm}$, and processed using Adobe Photoshop 9.0.2.

FACS and analysis. Splenic cell suspensions were obtained by manual smashing of total spleens; BM cell suspensions were obtained by flushing of multiple bones (femur, tibia, humerus, ulna) in sterile staining buffer (PBS containing $2 \%$ FCS). Cell surface stainings were performed in staining buffer for $30 \mathrm{~min}$, on ice, in the dark, with the appropriate combinations of saturating concentrations of the following conjugated monoclonal antibodies (mAbs) obtained from either BD Biosciences (San Jose, CA, USA), eBioscience (San Diego, CA, USA) or Biolegend (San Diego, CA, USA): CD71 (C2), TER-119 (Ly-76), CD11b (M1/70), CD45 (30-F11), CD49e (5H10-27, CD51 (RMV-7), CD61 (2C9.G2), CD29 (HMb11). F4/80 (A3-1) was purchased from Serotech (Kidlington, Oxford, UK). All macrophages sorting (FACS) experiments have been performed with the exclusion of SSChigh eosinophil population. Anti-Agrin antibody (Agr 247) was purchased from AbCam and its isotype control IgG1 from Invitrogen (Carlsbad, CA, USA); both antibodies were labeled using Zenon labeling technology (Molecular Probes). Anti$\alpha$-DG antibody $1 \mathrm{H} 6 \mathrm{C} 4$ or isotype control were purchased from Millipore and Biolegend, respectively. Rabbit anti-Integrin Beta 1/CD29 receptor phospho-specific (THR788/789) antibody (AB1929) was purchased from Millipore. Goat anti-mouseAlexa 647 and Goat anti-rabbit-Alexa 647, purchased from Life Technologies (Carlsbad, CA, USA), were used as secondary antibodies. For actin polimerization assay, sorted R2 cells were treated using Cytofix/Cytoperm Kit (BD Bioscience) according to the manufacturer's instructions and then stained with Phalloidin Alexa488 purchased from Life Technologies. For Annexin-V staining, freshly isolated cells were first stained with the appropriate $\mathrm{mAbs}$, and then washed in Annexin- $\mathrm{V}$ binding buffer (BD Biosciences) and incubated with Annexin-V (BD Biosciences). Dead cells were excluded using 7AAD or by low angle and orthogonal light scatter. The absolute count of leukocytes was performed using TruCount tubes (BD Biosciences) according to the manufacturer's instructions. Stained cells were analyzed with a FACSCanto I (2 lasers) or FACSCanto II (3 lasers) or LSR Fortessa (4 lasers) flow cytometer (BD Biosciences). Cell sorting experiments were performed using a FACSAria III (4 lasers; BD Biosciences).

Erythroblastic islands. Clusters composed of splenic macrophages and erythroblasts were isolated as already described. ${ }^{33}$ Bone marrow or splenic cell suspensions were allowed to attach to glass coverslips for $4 \mathrm{~h} 37^{\circ} \mathrm{C}$ in $5 \% \mathrm{CO}_{2}$. In all the reconstituted island experiments, an equal number of sorted control or 
Musk-LAgrn $^{-1-}$ Ter119+ erythroid cells were added to F4/80 $0^{+}$cells (i.e., 10 Ter $119^{+}$ cells per $\mathrm{F} 4 / 80^{+}$cell). When indicated, cells were incubated with anti- $\alpha$-DG antibody IIH6C4 (10 ug/ml, Millipore) or isotype control (Biolegend). Cells were fixed with formalin $(4 \%)$, blocked in $\mathrm{PBS} / 1 \%$ bovine serum albumin and incubated with the indicated antibodies. Confocal $z$ axis stacks were acquired and the number of TER-119-positive cells attached to each F4/80-positive, adherent macrophage was counted in $3 \mathrm{D}$ reconstructions.

Mouse phospho-RTK array. Murine sorted ctrl R2 cells $\left(5 \times 10^{6}\right)$ were stimulated or not with recombinant agrin ( $10 \mu \mathrm{g} / \mathrm{ml}$, R\&D Biosystems) for 5-30 min at $37{ }^{\circ} \mathrm{C}$, after pretreatment with anti- $\alpha$-DG blocking antibody $(10 \mu \mathrm{g} / \mathrm{ml}$, clone $\mathrm{IH} 6 \mathrm{CC} 4$, Millipore) when required. Cells were lysed, proteins were extracted, RTK membranes were probed and densitometric analyses were performed according to manufacturer's instructions (R\&D Systems, Catalog number ARY014).

Western blot analysis. K562 human erytholeukemic cell line maintained in RPMI 1640 medium (HEPES modification), with $10 \%$ fetal calf serum, $1 \%$ penicillin/ streptomycin, $2 \mathrm{mM} \mathrm{L}$-glutamine at $37^{\circ} \mathrm{C}, 5 \% \mathrm{CO} 2$ were seeded at $0.2 \times 10^{6} \mathrm{cells} /$ $\mathrm{ml}$ for $24 \mathrm{~h}$ prior to agrin treatment. Cells were lysed in 1\% Triton X-100, $20 \mathrm{mM}$ Tris- $\mathrm{HCl} \mathrm{pH} \mathrm{8,} 137 \mathrm{mM} \mathrm{NaCl}, 10 \%$ glycerol, $2 \mathrm{mM}$ EDTA, $1 \mathrm{mM}$ PMSF, $1 \mathrm{mM}$ $\mathrm{Na}_{3} \mathrm{VO}_{4}, 50 \mathrm{mM} \mathrm{NaF}, 10 \mathrm{mM} \mathrm{NaPP}, 5 \mu \mathrm{g} / \mathrm{ml}$ leupeptin, $5 \mu \mathrm{g} / \mathrm{ml}$ Aprotinin, containing protease inhibitors cocktail (Roche, Mannheim, Germany), with rotation at $4{ }^{\circ} \mathrm{C}$ for $15 \mathrm{~min}$. DNA complexes were disrupted incubating with Benzonase nuclease (Novagen, Darmstadt, Germany) for $10 \mathrm{~min}$ at room temperature. Proteins $(15 \mu \mathrm{g})$ after quantification by the BCA protein assay (Thermo Scientific, Pittisburgh, PA, USA) were resuspended in Laemmli sample buffer, separated by electrophoresis on 4-15\% TGX Stain-Free precast gels (Bio-Rad, Hercules, CA, USA) and transferred on PVDF membranes (Bio-Rad). The use of Stain-Free imaging allowed the normalization of bands to the total protein loaded on the gel, without housekeeping protein normalization or stripping and reprobing. Blots were probed with the primary antibody anti-DG (phospho Y893) or anti-Eph-B1 (phospho Y317) purchased from AbCam, according to the manufacturer's protocol and then with peroxidaseconjugated secondary antibodies (Bio-Rad). Blots were developed with Luminata Forte Western HRP substrate (Millipore) on the ChemiDoc MP System (Bio-Rad).

Immunoprecipitation. The human K562 erythroleukemia cell line was used for immunoprecipitation experiments. Cells were maintained in RPMI 1640 medium with $10 \%$ fetal calf serum, $1 \%$ penicillin/streptomycin, $2 \mathrm{mM} \mathrm{L}$-glutamine at $37^{\circ} \mathrm{C}$, $5 \% \mathrm{CO}_{2}$ and seeded at $0.2 \times 10^{6} \mathrm{celll} / \mathrm{ml}$ the day prior to treatment. Cells were resuspended in RPMI 1640 medium with $2 \%$ fetal calf serum and were stimulated or not with recombinant agrin $\left(10 \mu \mathrm{g} / \mathrm{ml}\right.$, R\&D Systems) for $10 \mathrm{~min}$ at $37^{\circ} \mathrm{C}$, after pretreatment with anti- $\alpha$-DG blocking antibody (10 $\mu \mathrm{g} / \mathrm{ml}$, clone $\mathrm{IHH} 6 \mathrm{C} 4$, Millipore) when required. Cells were lysed on ice for $30 \mathrm{~min}$ in $50 \mathrm{mM}$ Tris- $\mathrm{HCl}, \mathrm{pH} 7.4,2 \%$ CHAPS, protease inhibitor cocktail (Roche) and incubated with $375 \mathrm{U} / \mathrm{ml}$ Benzonase Nuclease (Merck Millipore, Massachusetts, USA). Magnetic beads with coupled sheep anti-mouse IgG (Dynabeads; Life Technologies) were washed with $0.1 \%$ bovine serum albumin IgG-free (Sigma-Aldrich, St. Louis, MO, USA) in PBS. Beads were incubated for $2 \mathrm{~h}$ at $4{ }^{\circ} \mathrm{C}$ with anti-EphB1 (clone 5F10A4, Thermo Fisher Scientific, Pittsburgh, PA USA). Equal amounts of protein $(1000 \mu \mathrm{g})$ were pre-cleared incubated overnight at $4{ }^{\circ} \mathrm{C}$ with primary antibody cross-linked to the beads. Cross-linking was carried out by incubation with $20 \mathrm{mM}$ dimethyl pimelimidate dihydrochloride (Sigma-Aldrich) in $0.2 \mathrm{M}$ triethanolamine $\mathrm{pH} 8.2$ for $30 \mathrm{~min}$ at room temperature. As a control, murine IgG-coated beads were used in each experiment. IPed proteins were eluted in Laemmli sample buffer. Samples were separated by 4-15\% TGX Stain-Free precast gels (Bio-Rad) and WB for DG (phospho Y893, AbCam) was performed. IP experiments were repeated several times on independent sample sets, with consistent results.

Statistical analysis. Statistical significance was calculated using the unpaired Student's t-test; (GraphPad Prism version 4.0). Differences were considered significant when $P \leqslant 0.05$ (asterisk), very significant when $P \leqslant 0.01$ (two asterisks) and extremely significant when $P \leqslant 0.001$ (three asterisks).

\section{Conflict of Interest}

The authors declare no conflict of interest.
Acknowledgements. We thank Francesco Piazza and Marco Sandri for critical reading of the MS, Steven Burden for providing mice, Chiara Buracchi and Paolo Somma for cell sorting experiments and Annarita Destro for assistance. This work was funded by the ERC AdG STePS to AV.

\section{Author contributions}

$\mathrm{AA}$ and $\mathrm{CM}$ conceived and designed the study and performed experiments; EL performed biochemical experiments; CS and RA performed confocal microscopy experiments; $\mathrm{CP}$ performed in vivo experiments; $\mathrm{GD}$ and $\mathrm{AS}$ provided suggestions and reagents; $\mathrm{CM}$ and $\mathrm{AV}$ supervised the study and wrote the manuscript; AV provided funds.

1. Bohme B, VandenBos T, Cerretti DP, Park LS, Holtrich U, Rubsamen-Waigmann $\mathrm{H}$ et al. Cell-cell adhesion mediated by binding of membrane-anchored ligand LERK-2 to the EPHrelated receptor human embryonal kinase 2 promotes tyrosine kinase activity. J Biol Chem 1996; 271: 24747-24752.

2. Wang HU, Anderson DJ. Eph family transmembrane ligands can mediate repulsive guidance of trunk neural crest migration and motor axon outgrowth. Neuron 1997; 18: 383-396.

3. Palmer A, Klein R. Multiple roles of ephrins in morphogenesis, neuronal networking, and brain function. Genes Dev 2003; 17: 1429-1450.

4. Zhao C, Irie N, Takada Y, Shimoda K, Miyamoto T, Nishiwaki T et al. Bidirectional ephrinB2EphB4 signaling controls bone homeostasis. Cell Metab 2006; 4: 111-121.

5. Wang Z, Miura N, Bonelli A, Mole P, Carlesso N, Olson DP et al. Receptor tyrosine kinase, EphB4 (HTK), accelerates differentiation of select human hematopoietic cells. Blood 2002; 99: 2740-2747.

6. Pasquale EB. Eph receptor signalling casts a wide net on cell behaviour. Nat Rev Mol Cell Biol 2005; 6: 462-475.

7. Dzierzak E, Philipsen S. Erythropoiesis: development and differentiation. Cold Spring Harb Perspect Med 2013; 3: a011601.

8. Bessis M. Erythroblastic island, functional unity of bone marrow. Rev Hematol 1958; 13: 8-11.

9. Manwani D, Bieker JJ. The erythroblastic island. Curr Top Dev Biol 2008; 82: 23-53.

10. McGrath KE. Red cell island dances: switching hands. Blood 2014; 123: 3847-3848.

11. Rosemblatt M, Vuillet-Gaugler MH, Leroy $C$, Coulombel L. Coexpression of two fibronectin receptors, VLA-4 and VLA-5, by immature human erythroblastic precursor cells. J Clin Invest 1991; 87: 6-11.

12. Gifford SC, Derganc J, Shevkoplyas SS, Yoshida T, Bitensky MW. A detailed study of timedependent changes in human red blood cells: from reticulocyte maturation to erythrocyte senescence. Br J Haematol 2006; 135: 395-404.

13. Lee G, Lo A, Short SA, Mankelow TJ, Spring F, Parsons SF et al. Targeted gene deletion demonstrates that the cell adhesion molecule ICAM-4 is critical for erythroblastic island formation. Blood 2006; 108: 2064-2071.

14. DeChiara TM, Bowen DC, Valenzuela DM, Simmons MV, Poueymirou WT, Thomas S et al. The receptor tyrosine kinase MuSK is required for neuromuscular junction formation in vivo. Cell 1996; 85: 501-512.

15. Mazzon C, Anselmo A, Cibella J, Soldani C, Destro A, Kim N et al. The critical role of agrin in the hematopoietic stem cell niche. Blood 2011; 118: 2733-2742.

16. Mazzon C, Anselmo A, Soldani $C$, Cibella J, Ploia $C$, Moalli $F$ et al. Agrin is required for survival and function of monocytic cells. Blood 2012; 119: 5502-5511.

17. Gautam M, Noakes PG, Moscoso L, Rupp F, Scheller RH, Merlie JP et al. Defective neuromuscular synaptogenesis in agrin-deficient mutant mice. Cell 1996; 85: 525-535.

18. Kim N, Burden SJ. MuSK controls where motor axons grow and form synapses. Nat Neurosci 2008; 11: 19-27.

19. Chow A, Huggins M, Ahmed J, Hashimoto D, Lucas D, Kunisaki Y et al. CD169(+) macrophages provide a niche promoting erythropoiesis under homeostasis and stress. Nat Med 2013; 19: 429-436.

20. Ramos P, Casu C, Gardenghi S, Breda L, Crielaard BJ, Guy E et al. Macrophages support pathological erythropoiesis in polycythemia vera and beta-thalassemia. Nat Med 2013; 19 : 437-445.

21. Chen K, Liu J, Heck S, Chasis JA, An X, Mohandas N. Resolving the distinct stages in erythroid differentiation based on dynamic changes in membrane protein expression during erythropoiesis. Proc Natl Acad Sci USA 2009; 106: 17413-17418.

22. Zhang W, Coldefy AS, Hubbard SR, Burden SJ. Agrin binds to the N-terminal region of Lrp4 protein and stimulates association between Lrp4 and the first immunoglobulin-like domain in muscle-specific kinase (MuSK). J Biol Chem 2011; 286: 40624-40630.

23. Davis S, Gale NW, Aldrich TH, Maisonpierre PC, Lhotak V, Pawson T et al. Ligands for EPHrelated receptor tyrosine kinases that require membrane attachment or clustering for activity. Science 1994; 266: 816-819.

24. Stein E, Lane AA, Cerretti DP, Schoecklmann HO, Schroff AD, Van Etten RL et al. Eph receptors discriminate specific ligand oligomers to determine alternative signaling complexes, attachment, and assembly responses. Genes Dev 1998; 12: 667-678.

25. Zantek ND, Azimi M, Fedor-Chaiken M, Wang B, Brackenbury R, Kinch MS. E-cadherin regulates the function of the EphA2 receptor tyrosine kinase. Cell Growth Differ 1999; 10: 629-638. 
26. Yuan ZL, Guan YJ, Wang L, Wei W, Kane AB, Chin YE. Central role of the threonine residue within the $p+1$ loop of receptor tyrosine kinase in STAT3 constitutive phosphorylation in metastatic cancer cells. Mol Cell Biol 2004; 24: 9390-9400.

27. Pribiag H, Peng H, Shah WA, Stellwagen D, Carbonetto S. Dystroglycan mediates homeostatic synaptic plasticity at GABAergic synapses. Proc Natl Acad Sci USA 2014; 111 6810-6815.

28. Huynh-Do U, Stein E, Lane AA, Liu H, Cerretti DP, Daniel TO. Surface densities of ephrin-B1 determine EphB1-coupled activation of cell attachment through alphavbeta3 and alpha5beta1 integrins. EMBO J 1999; 18: 2165-2173.

29. Hanspal M, Hanspal JS. The association of erythroblasts with macrophages promotes erythroid proliferation and maturation: a $30-\mathrm{kD}$ heparin-binding protein is involved in this contact. Blood 1994; 84: 3494-3504.

30. Hanspal M, Smockova Y, Uong Q. Molecular identification and functional characterization of a novel protein that mediates the attachment of erythroblasts to macrophages. Blood 1998; 92: $2940-2950$.

31. Sadahira Y, Yoshino T, Monobe Y. Very late activation antigen 4-vascular cell adhesion molecule 1 interaction is involved in the formation of erythroblastic islands. J Exp Med 1995; 181: 411-415.
32. Martin PT, Sanes JR. Integrins mediate adhesion to agrin and modulate agrin signaling. Development 1997; 124: 3909-3917.

33. lavarone A, King ER, Dai XM, Leone G, Stanley ER, Lasorella A. Retinoblastoma promotes definitive erythropoiesis by repressing Id2 in fetal liver macrophage. Nature 2004; 432(7020): 1040-1045.

(c) (i) (2) $\odot$ This work is licensed under a Creative Commons Attribution-NonCommercial-NoDerivs 4.0 International License. The images or other third party material in this article are included in the article's Creative Commons license, unless indicated otherwise in the credit line; if the material is not included under the Creative Commons license, users will need to obtain permission from the license holder to reproduce the material. To view a copy of this license, visit http://creativecommons.org/licenses/by-nc-nd/4.0/

Supplementary Information accompanies this paper on Cell Death and Differentiation website (http://www.nature.com/cdd) 